\title{
The persistent problem of new-onset postoperative atrial fibrillation: A single-institution experience over two decades
}

\author{
Jeanne Shen, BS, Shelly Lall, MD, Victoria Zheng, MD, Patricia Buckley, RHIT, \\ Ralph J. Damiano, Jr, MD and Richard B. Schuessler, PhD
}

\begin{abstract}
Objective: Postoperative atrial fibrillation is the most common complication after cardiac surgery. A variety of postoperative atrial fibrillation risk factors have been reported, but study results have been inconsistent or contradictory, particularly in patients with preexisting atrial fibrillation. The incidence of postoperative atrial fibrillation was evaluated in a group of 10,390 patients undergoing cardiac surgery among a comprehensive range of risk factors to identify reliable predictors of postoperative atrial fibrillation.
\end{abstract}

\begin{abstract}
Methods: This 20-year retrospective study examined the relationship between postoperative atrial fibrillation and demographic factors, preoperative health conditions and medications, operative procedures, and postoperative complications. Multivariate logistic regression models were used to evaluate potential predictors of postoperative atrial fibrillation.

Results: Increasing age, mitral valve surgery (odds ratio $=1.91$ ), left ventricular aneurysm repair (odds ratio $=1.57)$, aortic valve surgery (odds ratio $=1.52$ ), race (Caucasian) (odds ratio $=1.51)$, use of cardioplegia (odds ratio $=1.36$ ), use of an intraaortic balloon pump (odds ratio $=1.28$ ), previous congestive heart failure (odds ratio $=1.28$ ), and hypertension (odds ratio $=1.15$ ) were significantly associated with postoperative atrial fibrillation. The non-linear relationship between age and postoperative atrial fibrillation revealed the acceleration of postoperative atrial fibrillation risk in patients aged 55 years or more. In patients undergoing coronary artery bypass grafting, increasing age and previous congestive heart failure were the only factors associated with a higher risk of postoperative atrial fibrillation. There was no trend in incidence of postoperative atrial fibrillation over time. No protective factors against postoperative atrial fibrillation were detected, including commonly prescribed categories of medications.
\end{abstract}

Conclusions: The persistence of the problem of postoperative atrial fibrillation and the modest predictability using common risk factors suggest that limited progress has been made in understanding its cause and treatment. (J Thorac Cardiovasc Surg 2011;141:559-70)

Postoperative atrial fibrillation (POAF) is the most common complication after cardiac surgery. POAF occurs early in the recovery period after surgery, is associated with an increased incidence of mortality and other morbidities, and contributes significantly to longer hospital stays and higher cost of care. ${ }^{1-4}$ The reported incidence ranges from $11 \%$ to $50 \%$, depending on the patient cohort evaluated. ${ }^{1}$ Because the underlying pathophysiologic mechanisms responsible for POAF remain largely undetermined, the use of statistical models to identify potential predictors of its occurrence is

\footnotetext{
From the Barnes-Jewish Hospital and Washington University in Saint Louis, School of Medicine, Division of Cardiothoracic Surgery, St Louis, Mo.

This study was supported by National Institutes of Health (NIH) Training Grant 2T35HL007815-11A1 and Grants NIH R01 HL 032257, NIH R01 HL085113, and NIH T32 HL007776.

Disclosures: Authors have nothing to disclose with regard to commercial support

Received for publication Aug 6, 2009; revisions received Feb 8, 2010; accepted for publication March 9, 2010; available ahead of print May 3, 2010.

Address for reprints: Richard B. Schuessler, PhD, Division of Cardiothoracic Surgery, 660 South Euclid, Campus Box 8234, Washington University School of Medicine, St Louis, MO 63110 (E-mail: schuesslerr@wustl.edu).

$0022-5223 / \$ 36.00$

Copyright $(c) 2011$ by The American Association for Thoracic Surgery

doi:10.1016/j.jtcvs.2010.03.011
}

the most practical approach to POAF prevention and evaluation of potential treatments. Various risk factors for POAF have been reported; however, study results have been inconsistent or contradictory, possibly because of small study sample sizes, limited control of confounding factors, and interference from preexisting atrial fibrillation. Only increasing age has been a consistently reported independent risk factor for POAF. ${ }^{2,45}$ Evaluating predictors of POAF across studies has also been difficult because the characteristics of study samples are rarely replicable, and confounding risk factors differ between studies. This may lead to biased or limited generalization to the overall patient population undergoing cardiac surgery.

In the 20-year period from 1986 to 2005, 14,960 cardiac surgical procedures were performed at Barnes-Jewish Hospital. This large patient population provided a unique opportunity for a comprehensive study of POAF. The primary objectives of this 20 -year retrospective study were to evaluate trends in the incidence of POAF over time and potential predictors of POAF. Various cardiovascular medications are frequently administered to patients as prophylaxis against postoperative arrhythmias. However, the data supporting these practices have been inconsistent. This study also 


\section{Abbreviations and Acronyms \\ AUC = area under the receiver operating characteristic curve \\ $\mathrm{CABG}=$ coronary artery bypass grafting \\ $\mathrm{CHF}=$ congestive heart failure \\ MLR = multivariate logistic regression \\ $\mathrm{OR}=$ odds ratio \\ POAF $=$ postoperative atrial fibrillation}

sought to evaluate the effects of various cardiovascular and non-cardiovascular medications on the incidence of POAF when administered preoperatively. An additional objective of the study was to evaluate the association between POAF and postoperative outcomes.

\section{MATERIALS AND METHODS \\ Study Population}

From January 1, 1986, to December 30, 2005, 14,960 cardiac surgical procedures were performed at Barnes-Jewish Hospital. Of these cases, 10,390 patients without preexisting atrial arrhythmias (atrial fibrillation, atrial flutter, and paroxysmal atrial tachycardia) were selected for inclusion in this study. Appropriate institutional review board approval was obtained.

Patient ages ranged from 12 to 94 years (median $=64$ years), with a mean of $62.3 \pm 12.9$ years. Men comprised $65 \%$ of the study population, and more than $87 \%$ of the patients were Caucasian. Approximately $79 \%$ of the procedures were elective, with the remaining being emergency $(11 \%)$ or urgent $(10 \%)$ operations. After surgery, all patients received continuous 24-hour telemetry with arrhythmia-detection algorithms during their entire hospital stay.

\section{Measures}

Patient information, including demographics, preoperative medical history, and peri- and postoperative data, was extracted from individual medical records. The onset of POAF was the dichotomous dependent variable in the analyses. POAF was diagnosed as atrial fibrillation/flutter occurring during the postoperative recovery period before hospital discharge and requiring treatment. This did not include transient, nonsustained arrhythmias, or arrhythmias treated only with oxygen or potassium supplementation. Fiftyone independent variables, or potential predictors, were organized into 4 groups: (1) demographics, (2) preoperative medical history, (3) preoperative medications, and (4) intraoperative procedures (type of surgery performed and any other procedures or devices used). A complete list of independent variables is included in Table 1. It should be noted that, although one of the aims of this study was to evaluate the efficacy of various medications as POAF prophylaxis, the reason for administering a particular medication at the time may have been POAF prophylaxis, or a different reason altogether, such as an acute or chronic condition.

Information on postoperative complications, such as stroke, length of hospital stay after surgery, and mortality, was also collected (listed in Table 2) to look for any association with new-onset POAF.

\section{Analysis}

Descriptive statistics, chi-square tests, Mann-Whitney Wilcoxon tests, and odds ratios (ORs) were applied to describe the study population and the univariate relationships between individual variables and POAF.

Multivariate logistic regression (MLR) models were used to predict the onset of POAF, with a stepwise selection of significant predictors. Because of the large size of the study population, a stringent selection criterion of a $P$ value of .05 or less was used for entering and removing independent variables in the model. In a large-scale database with more than 50 potential predictors, the presence of missing data is inevitable; its cumulative impact on multivariate analyses due to case-wise deletion often overwhelmingly reduces the number of patients available for analysis. To minimize this impact, the MLR incorporated only those independent variables that were significant at the $P$ level of .10 or less after univariate analysis. The onset of POAF was predicted by MLR for all patients undergoing cardiac surgery (model 1) and for patients who only underwent coronary artery bypass grafting (CABG) (model 2). Because of case-wise deletion caused by missing values, model 1 had only 5075 patients available for analysis, which yielded 10 significant predictors of POAF. By using only these 10 significant predictors in a third MLR model (model 3), we were able to retain more cases $(\mathrm{N}=10,371)$ while verifying the stability of these 10 predictors when reapplied to the entire study population (all patients undergoing cardiac surgery).

Information on preoperative warfarin use was missing for 5354 patients. To avoid case-wise deletion of these cases in the comprehensive analysis, this variable was excluded from the MLR models. In a preliminary MLR model, warfarin use was coded as “yes,", "no,", or " unknown', to retain missing cases in the analysis. This showed that preoperative warfarin use was not significantly different from no use in terms of predicting POAF. Therefore, it was safe to exclude this variable from the final analyses with minimal risk of losing a predictor of POAF.

Analyses were conducted using SAS software, version 9.1. ${ }^{6}$ Three statistics were calculated to evaluate the appropriateness of the models: the Hosmer-Lemeshow statistic testing the goodness of fit between the observed and the predicted occurrence of POAF; the likelihood ratio testing the global significance of the selected predictors of POAF; and the area under the receiver operating characteristic curve (AUC) depicting the adequacy of the model for detecting the onset of POAF.

\section{RESULTS}

The average incidence of POAF was found to be $30 \%$ (range, $25 \%-36 \%$ ) for the study population over the 20 year period (Figure 1). There was no significant effect of year of surgery on POAF $(P=.17)$. The incidence of POAF ranged from $14 \%$ to $47 \%$ across 4 different age groups (Table 1). The odds of having POAF in patients aged 72 years or more were 5.6 times $(\mathrm{OR}=5.57)$ greater than in patients aged 12 to 54 years. Although increasing age has consistently been associated with a higher incidence of POAF, the relationship between age and POAF is nonlinear. Figure 2 illustrates that beyond age 55 years, for example, the estimated probability of having POAF increases more steeply, from $20 \%$ to $80 \%$, whereas the probability increases more slowly (up to $20 \%$ ) for patients aged less than 55 years.

Table 1 presents the results of univariate analysis of the association between individual variables and new-onset POAF. ORs and their $95 \%$ confidence intervals are presented for those variables that were significantly related to POAF. Caucasian patients had $59 \%$ greater odds $(\mathrm{OR}=1.59)$ of having POAF compared with patients of African-American descent. The likelihoods of developing POAF in patients with a preoperative history of congestive heart failure (CHF), chronic obstructive pulmonary disease, hypertension, or aortic valve stenosis were approximately 1.6, 1.4, 1.3, and 1.7 times, respectively, those in patients without such corresponding histories. Similarly, the incidence of POAF in patients 
TABLE 1. Chi-square and odds ratios for univariate predictors and associates of postoperative atrial fibrillation

\begin{tabular}{|c|c|c|c|c|c|}
\hline \multirow[b]{2}{*}{ Variable* } & \multicolumn{2}{|c|}{ POAF } & \multicolumn{2}{|c|}{ Chi-square test } & \multirow{2}{*}{$\begin{array}{c}\text { Odds ratio } \\
\text { OR }(95 \% \text { CI })\end{array}$} \\
\hline & No, n $(\%)$ & Yes, n (\%) & $\chi^{2}$ & $P$ & \\
\hline \multicolumn{6}{|l|}{ Demographics } \\
\hline Quartile age group & & & 766.57 & $<.0001$ & \\
\hline $12 \sim 54 \mathrm{y}$ & $2321(86 \%)$ & $364(14 \%)$ & & & ref \\
\hline $55 \sim 63 \mathrm{y}$ & $1888(75 \%)$ & $624(25 \%)$ & & & $2.11(1.83-2.43)$ \\
\hline $64 \sim 71 \mathrm{y}$ & $1646(64 \%)$ & $945(36 \%)$ & & & $3.66(3.19-4.19)$ \\
\hline $72+y$ & $1389(53 \%)$ & $1213(47 \%)$ & & & $5.57(4.86-6.37)$ \\
\hline Gender & & & 0.33 & .5650 & \\
\hline Female & $2541(69 \%)$ & $1122(31 \%)$ & & & \\
\hline Male & $4703(70 \%)$ & $2024(30 \%)$ & & & \\
\hline Race & & & 43.26 & $<.0001$ & \\
\hline African-American & $921(78 \%)$ & $266(22 \%)$ & & & ref \\
\hline Caucasian & $6205(69 \%)$ & $2841(31 \%)$ & & & $1.59(1.37-1.83)$ \\
\hline Other & $107(76 \%)$ & $33(24 \%)$ & & & $1.07(0.71-1.61)$ \\
\hline \multicolumn{6}{|l|}{ Preoperative medical history } \\
\hline Smoking history & & & 11.60 & .0007 & \\
\hline No & $2292(67 \%)$ & $1109(33 \%)$ & & & ref \\
\hline Yes & $4866(71 \%)$ & $2019(29 \%)$ & & & $0.86(0.78-0.94)$ \\
\hline Current smoker & & & 66.53 & $<.0001$ & \\
\hline No & $5201(67 \%)$ & $2510(33 \%)$ & & & ref \\
\hline Yes & $1956(76 \%)$ & $618(24 \%)$ & & & $0.65(0.59-0.73)$ \\
\hline COPD & & & 29.99 & $<.0001$ & \\
\hline No & $6431(71 \%)$ & $2676(29 \%)$ & & & ref \\
\hline Yes & $813(63 \%)$ & $470(37 \%)$ & & & $1.39(1.23-1.57)$ \\
\hline Hypertension & & & 35.90 & $<.0001$ & \\
\hline No & $2750(73 \%)$ & $1001(27 \%)$ & & & ref \\
\hline Yes & $4494(68 \%)$ & $2145(32 \%)$ & & & $1.31(1.20-1.43)$ \\
\hline Angina pectoris & & & 12.30 & .0021 & \\
\hline None & $2048(69 \%)$ & $923(31 \%)$ & & & ref \\
\hline Stable & $2357(72 \%)$ & $915(28 \%)$ & & & $0.86(0.77-0.96)$ \\
\hline Unstable & $2839(68 \%)$ & $1308(32 \%)$ & & & $1.02(0.92-1.13)$ \\
\hline Myocardial infarction & & & 4.29 & .0384 & \\
\hline No & $3828(71 \%)$ & $1593(29 \%)$ & & & ref \\
\hline Yes & $3416(69 \%)$ & $1553(31 \%)$ & & & $1.09(1.00-1.19)$ \\
\hline $\mathrm{CHF}$ & & & 94.49 & $<.0001$ & \\
\hline No & $5365(73 \%)$ & $2034(27 \%)$ & & & ref \\
\hline Yes & $1878(63 \%)$ & $1111(37 \%)$ & & & $1.56(1.43-1.71)$ \\
\hline NYHA score for CHF & & & 105.75 & $<.0001$ & \\
\hline 0 & $3304(74 \%)$ & $1166(26 \%)$ & & & ref \\
\hline 1 & $20(61 \%)$ & $13(39 \%)$ & & & $1.84(0.91-3.72)$ \\
\hline 2 & $440(62 \%)$ & $274(38 \%)$ & & & $1.77(1.50-2.08)$ \\
\hline 3 & $687(62 \%)$ & $417(38 \%)$ & & & $1.72(1.50-1.98)$ \\
\hline 4 & $730(64 \%)$ & $407(36 \%)$ & & & $1.58(1.38-1.81)$ \\
\hline Stroke & & & 5.47 & .0194 & \\
\hline No & $6648(70 \%)$ & $2843(30 \%)$ & & & ref \\
\hline Yes & $596(66 \%)$ & $303(31 \%)$ & & & $1.19(1.03-1.37)$ \\
\hline Peripheral vascular disease & & & 19.44 & $<.0001$ & \\
\hline No & $5201(67 \%)$ & $2510(33 \%)$ & & & ref \\
\hline Yes & $1956(76 \%)$ & $618(24 \%)$ & & & $1.29(1.15-1.44)$ \\
\hline Diabetes & & & 0.93 & .3343 & \\
\hline No & $5168(70 \%)$ & $2215(30 \%)$ & & & \\
\hline Yes & $2076(69 \%)$ & $931(31 \%)$ & & & \\
\hline Renal insufficiency & & & 9.75 & .0018 & \\
\hline No & $6653(70 \%)$ & $2834(30 \%)$ & & & ref \\
\hline Yes & $576(65 \%)$ & $309(35 \%)$ & & & $1.26(1.09-1.46)$ \\
\hline
\end{tabular}


TABLE 1. Continued

\begin{tabular}{|c|c|c|c|c|c|}
\hline \multirow[b]{2}{*}{ Variable* } & \multicolumn{2}{|c|}{ POAF } & \multicolumn{2}{|c|}{ Chi-square test } & \multirow{2}{*}{$\begin{array}{c}\text { Odds ratio } \\
\text { OR }(95 \% \text { CI }) \\
\end{array}$} \\
\hline & No, n $(\%)$ & Yes, n $(\%)$ & $\chi^{2}$ & $P$ & \\
\hline Dialysis & & & 0.16 & .6854 & \\
\hline No & $7112(70 \%)$ & $3085(30 \%)$ & & & \\
\hline Yes & $132(68 \%)$ & $61(32 \%)$ & & & \\
\hline Aortic valve insufficiency & & & 16.55 & .0003 & \\
\hline None/mild & $5644(69 \%)$ & $2420(31 \%)$ & & & ref \\
\hline Moderate & $242(62 \%)$ & $151(38 \%)$ & & & $1.42(1.15-1.75)$ \\
\hline Severe & $263(64 \%)$ & $151(36 \%)$ & & & $1.31(1.06-1.60)$ \\
\hline Mitral valve insufficiency & & & 32.84 & $<.0001$ & \\
\hline None/mild & $5435(70 \%)$ & $2306(30 \%)$ & & & ref \\
\hline Moderate & $548(66 \%)$ & $281(34 \%)$ & & & $1.21(1.04-1.41)$ \\
\hline Severe & $537(61 \%)$ & $339(39 \%)$ & & & $1.49(1.29-1.72)$ \\
\hline Tricuspid valve insufficiency & & & 1.73 & .4214 & \\
\hline None/mild & $5824(69 \%)$ & $2600(31 \%)$ & & & \\
\hline Moderate & $215(67 \%)$ & $107(33 \%)$ & & & \\
\hline Severe & $92(73 \%)$ & $34(27 \%)$ & & & \\
\hline Aortic valve stenosis & & & 72.71 & $<.0001$ & \\
\hline No & $6550(71 \%)$ & $2672(29 \%)$ & & & ref \\
\hline Yes & $663(59 \%)$ & $467(41 \%)$ & & & $1.73(1.52-1.96)$ \\
\hline Mitral valve stenosis & & & 5.78 & .0162 & \\
\hline No & $7120(70 \%)$ & $3079(30 \%)$ & & & ref \\
\hline Yes & $95(61 \%)$ & $61(39 \%)$ & & & $1.48(1.07-2.05)$ \\
\hline Tricuspid valve stenosis & & & - & - & \\
\hline No & $7210(70 \%)$ & $3141(30 \%)$ & & & \\
\hline Yes & $2(100 \%)$ & $0(0 \%)$ & & & \\
\hline PCI & & & 6.38 & .0116 & \\
\hline No & $6184(71 \%)$ & $2578(29 \%)$ & & & ref \\
\hline Yes & $1060(65 \%)$ & $568(35 \%)$ & & & $0.86(0.76-0.97)$ \\
\hline Previous CABG & & & 1.47 & .2258 & \\
\hline No & $6705(70 \%)$ & $2933(30 \%)$ & & & \\
\hline Yes & $539(72 \%)$ & $213(28 \%)$ & & & \\
\hline Previous valve procedure & & & 4.90 & .0269 & \\
\hline No & $7083(70 \%)$ & $3097(30 \%)$ & & & ref \\
\hline Yes & $161(77 \%)$ & $49(23 \%)$ & & & $0.70(0.50-0.96)$ \\
\hline Previous LVA repair & & & 0.75 & .3859 & \\
\hline No & $7232(70 \%)$ & $3143(30 \%)$ & & & \\
\hline Yes & $12(80 \%)$ & $3(20 \%)$ & & & \\
\hline Congenital defect repair & & & 7.34 & .0067 & \\
\hline No & $7191(70 \%)$ & $3137(30 \%)$ & & & ref \\
\hline Yes & $53(85 \%)$ & $9(15 \%)$ & & & $0.39(0.19-0.79)$ \\
\hline \multicolumn{6}{|l|}{ Preoperative medications } \\
\hline Antiarrhythmics & & & 0.79 & .3729 & \\
\hline No & $4557(69 \%)$ & $2001(31 \%)$ & & & \\
\hline Yes & $146(67 \%)$ & $73(33 \%)$ & & & \\
\hline Beta-blockers & & & 1.42 & .2327 & \\
\hline No & $3657(69 \%)$ & $1631(31 \%)$ & & & \\
\hline Yes & $3556(70 \%)$ & $1507(30 \%)$ & & & \\
\hline $\mathrm{Ca}^{2+}$-channel blocker & & & 0.14 & .7119 & \\
\hline No & $4332(69 \%)$ & $1906(31 \%)$ & & & \\
\hline Yes & $435(70 \%)$ & $185(30 \%)$ & & & \\
\hline IV nitrates & & & 0.21 & .6488 & \\
\hline No & $6633(70 \%)$ & $2876(30 \%)$ & & & \\
\hline Yes & $581(69 \%)$ & $261(31 \%)$ & & & \\
\hline
\end{tabular}


TABLE 1. Continued

\begin{tabular}{|c|c|c|c|c|c|}
\hline \multirow[b]{2}{*}{ Variable* } & \multicolumn{2}{|c|}{ POAF } & \multicolumn{2}{|c|}{ Chi-square test } & \multirow{2}{*}{$\begin{array}{c}\text { Odds ratio } \\
\text { OR }(95 \% \text { CI })\end{array}$} \\
\hline & No, n $(\%)$ & Yes, $n(\%)$ & $\chi^{2}$ & $P$ & \\
\hline Digitalis & & & 3.84 & .0500 & \\
\hline No & $6207(70 \%)$ & $2624(30 \%)$ & & & ref \\
\hline Yes & $557(67 \%)$ & $274(33 \%)$ & & & $1.16(1.00-1.35)$ \\
\hline Inotropic agents & & & 0.22 & .6381 & \\
\hline No & $7039(70 \%)$ & $3056(30 \%)$ & & & \\
\hline Yes & $175(68 \%)$ & $81(32 \%)$ & & & \\
\hline ACE inhibitors & & & 1.36 & .2431 & \\
\hline No & $4235(69 \%)$ & $1868(31 \%)$ & & & \\
\hline Yes & $1639(68 \%)$ & $768(32 \%)$ & & & \\
\hline Diuretics & & & 22.27 & $<.0001$ & \\
\hline No & $5203(71 \%)$ & $2099(29 \%)$ & & & ref \\
\hline Yes & $1573(66 \%)$ & $805(34 \%)$ & & & $1.27(1.15-1.40)$ \\
\hline Aspirin/Ecotrin & & & 2.28 & .1314 & \\
\hline No & $2789(71 \%)$ & $1164(29 \%)$ & & & \\
\hline Yes & $4425(69 \%)$ & $1974(31 \%)$ & & & \\
\hline Coumadin & & & 5.32 & .0211 & \\
\hline No & $3214(68 \%)$ & $1535(32 \%)$ & & & ref \\
\hline Yes & $213(74 \%)$ & $74(26 \%)$ & & & $0.7(0.55-0.95)$ \\
\hline Anticoagulants (non-Coumadin) & & & 2.09 & .1479 & \\
\hline No & $2259(68 \%)$ & $1058(32 \%)$ & & & \\
\hline Yes & $3293(70 \%)$ & $1437(30 \%)$ & & & \\
\hline Lipid-lowering medications & & & 1.44 & .2301 & \\
\hline No & $3238(67 \%)$ & $1571(33 \%)$ & & & \\
\hline Yes & $575(69 \%)$ & $253(31 \%)$ & & & \\
\hline Steroids & & & 2.42 & .1196 & \\
\hline No & $7009(70 \%)$ & $3030(30 \%)$ & & & \\
\hline Yes & $205(66 \%)$ & $107(34 \%)$ & & & \\
\hline \multicolumn{6}{|l|}{ Intraoperative procedures } \\
\hline Clinical status & & & 3.07 & .2153 & \\
\hline Elective & $5695(70 \%)$ & $2448(30 \%)$ & & & \\
\hline Emergency & $797(70 \%)$ & $343(30 \%)$ & & & \\
\hline Urgent & $728(67 \%)$ & $353(33 \%)$ & & & \\
\hline Valve procedure & & & 109.28 & $<.0001$ & \\
\hline No & $5853(72 \%)$ & $2251(28 \%)$ & & & ref \\
\hline Yes & $1391(61 \%)$ & $895(39 \%)$ & & & $1.67(1.52-1.84)$ \\
\hline Aortic valve procedure & & & 80.27 & $<.0001$ & \\
\hline No & $6366(71 \%)$ & $2555(29 \%)$ & & & ref \\
\hline Yes & $878(60 \%)$ & $591(40 \%)$ & & & $1.68(1.50-1.88)$ \\
\hline Mitral valve procedure & & & 59.07 & $<.0001$ & \\
\hline No & $6699(71 \%)$ & $2762(29 \%)$ & & & ref \\
\hline Yes & $545(59 \%)$ & $384(41 \%)$ & & & $1.71(1.49-1.96)$ \\
\hline Tricuspid valve procedure & & & 3.70 & .0543 & \\
\hline No & $7164(70 \%)$ & $3124(30 \%)$ & & & \\
\hline Yes & $80(78 \%)$ & $22(22 \%)$ & & & \\
\hline CABG & & & 9.74 & .0018 & \\
\hline No & $1607(72 \%)$ & $612(28 \%)$ & & & ref \\
\hline Yes & $5637(69 \%)$ & $2534(31 \%)$ & & & $1.18(1.06-1.31)$ \\
\hline CABG and valve procedure & & & 204.05 & $<.0001$ & \\
\hline Neither & $667(81 \%)$ & $153(19 \%)$ & & & ref \\
\hline CABG only & $5186(71 \%)$ & $2098(29 \%)$ & & & $1.76(1.47-2.12)$ \\
\hline Valve only & $940(67 \%)$ & $459(33 \%)$ & & & $2.13(1.73-2.62)$ \\
\hline Both & $451(51 \%)$ & $436(49 \%)$ & & & $4.21(3.38-5.25)$ \\
\hline
\end{tabular}


TABLE 1. Continued

\begin{tabular}{|c|c|c|c|c|c|}
\hline \multirow[b]{2}{*}{ Variable* } & \multicolumn{2}{|c|}{ POAF } & \multicolumn{2}{|c|}{ Chi-square test } & \multirow{2}{*}{$\begin{array}{c}\text { Odds ratio } \\
\text { OR }(95 \% \text { CI })\end{array}$} \\
\hline & No, n $(\%)$ & Yes, n (\%) & $\chi^{2}$ & $\boldsymbol{P}$ & \\
\hline LVA repair & & & 9.13 & .0025 & \\
\hline No & $7124(70 \%)$ & $3066(30 \%)$ & & & ref \\
\hline Yes & $120(60 \%)$ & $80(40 \%)$ & & & $1.55(1.16-2.06)$ \\
\hline VSD repair & & & 2.92 & .0877 & \\
\hline No & $7228(70 \%)$ & $3133(30 \%)$ & & & \\
\hline Yes & $16(55 \%)$ & $13(45 \%)$ & & & \\
\hline Ventricular assist device & & & 0.19 & .6665 & \\
\hline No & $7167(70 \%)$ & $3110(30 \%)$ & & & \\
\hline Yes & $76(68 \%)$ & $36(32 \%)$ & & & \\
\hline Cardioplegia used & & & 41.86 & $<.0001$ & \\
\hline No & $805(79 \%)$ & $220(21 \%)$ & & & ref \\
\hline Yes & $6439(69 \%)$ & $2926(31 \%)$ & & & $1.66(1.42-1.94)$ \\
\hline IABP used & & & 34.62 & $<.0001$ & \\
\hline No & $6530(71 \%)$ & $2712(29 \%)$ & & & ref \\
\hline Yes & $714(62 \%)$ & $434(38 \%)$ & & & $1.46(1.29-1.66)$ \\
\hline Crossclamp time (min) & & & 27.54 & $<.0001$ & \\
\hline 0 & $515(75 \%)$ & $172(25 \%)$ & & & ref \\
\hline $3-49$ & $1733(72 \%)$ & $679(28 \%)$ & & & $1.17(0.97-1.42)$ \\
\hline $50-69$ & $1638(69 \%)$ & $724(31 \%)$ & & & $1.32(1.02-1.61)$ \\
\hline $70-97$ & $1654(69 \%)$ & $740(31 \%)$ & & & $1.34(1.10-1.63)$ \\
\hline $98-410$ & $1575(66 \%)$ & $800(34 \%)$ & & & $1.52(1.26-1.84)$ \\
\hline
\end{tabular}

$P O A F$, Postoperative atrial fibrillation; $O R$, odds ratio; $C I$, confidence interval; $C O P D$, chronic obstructive pulmonary disease; $C H F$, congestive heart failure; $N Y H A$, New York Heart Association; $C A B G$, coronary artery bypass graft; $L V A$, left ventricular aneurysm; $I V$, intravenous; $A C E$, angiotensin-converting enzyme; $V S D$, ventricular septal defect; $P C I$, percutaneous cardiac intervention; $I A B P$, intraaortic balloon pump. *The year when the current surgery was performed is not shown but was included in the multivariate analysis.

undergoing an aortic or mitral valve procedure was approximately 1.7 times that in patients undergoing other cardiac procedures.

Twelve of the 13 types of preoperative medications included in this study did not show a preventive effect against POAF, contrary to what has been reported for some of these medications in controlled trials ${ }^{7,8}$ or retrospective studies. ${ }^{1,9}$ During the 20-year period, the preoperative use of betablockers increased from $36 \%$ to $59 \%$. Despite this increased use, the incidence of POAF did not decrease. Overall, the incidence was $30 \%$ for those patients receiving beta-blockers, versus $31 \%$ for those who were not. Likewise, the use of angiotensin-converting enzyme inhibitors increased from $5 \%$ to $47 \%$ during the 20 -year period, with no significant reduction in POAF. Although the univariate analysis (Table 1) showed that warfarin reduced the chance of POAF by $30 \%$, this effect was not significant after controlling for other factors in the multivariate analysis. Similarly, patients taking digitalis or diuretics had a higher risk of POAF (Table 1), but this effect also did not hold up in the multivariate analysis.

Table 2 shows the co-occurrence of POAF and other postoperative complications, as well as the association between POAF and the length of hospital stay and mortality after cardiac procedures. POAF was positively associated with permanent stroke, the requirement for implantation of a permanent pacemaker, and mortality $(\mathrm{OR}=2.42$,
$\mathrm{OR}=2.62$, and $\mathrm{OR}=1.75$, respectively). Among the 14 complications studied other than POAF, 13 had a positive association with POAF (Table 2). The incidence of death from a neurologic cause was more than doubled in patients with POAF.

Consistent with the results of other studies, ${ }^{1-3}$ the occurrence of POAF was associated with a 2-day longer hospital stay, as evaluated in this study by the median length of stay from surgery to discharge and a 1-day longer median length of stay in the intensive care unit and stepdown unit after surgery (see Table 2 for details).

Table 3 presents significant variables as independent predictors of POAF that were detected by the MLR models. The goodness-of-fit, likelihood ratio, and AUC show that the models fit the data adequately.

For all types of cardiac procedures collectively (model 1), increasing age was strongly associated with POAF, as expected. The odds of a person aged $72+$ years developing POAF, for example, are 4.4 times those for a patient aged less than 55 years. Over the 20 -year period, the average patient age ranged from 60 to 64 years. There was no trend in age over the study period. The average age was $60 \pm 13$ years in 1986 and $61 \pm 14$ years in 2005. New-onset POAF was 1.5 times as likely to occur among Caucasians as among patients of African-American or other racial/ethnic backgrounds. A history of CHF was associated with a $40 \%$ higher risk of developing POAF, and preexisting hypertension was related 
TABLE 2. Association between new-onset postoperative atrial fibrillation and postoperative course $(\mathbf{N}=10,390)$

\begin{tabular}{|c|c|c|c|c|c|}
\hline \multirow[b]{2}{*}{ Variable } & \multicolumn{2}{|c|}{ POAF } & \multicolumn{2}{|c|}{ Chi-square test } & \multirow{2}{*}{$\begin{array}{c}\text { Odds ratio } \\
\text { OR }(95 \% \text { CI })\end{array}$} \\
\hline & No, n $(\%)$ & Yes, n (\%) & $\chi^{2}$ & $\boldsymbol{P}$ & \\
\hline \multicolumn{6}{|l|}{ Complication } \\
\hline MI & & & 8.82 & .0030 & \\
\hline No & $7201(70 \%)$ & $3110(30 \%)$ & & & ref \\
\hline Yes & $43(54 \%)$ & $895(46 \%)$ & & & $1.94(1.24-3.02)$ \\
\hline Permanent pacemaker & & & 50.10 & $<.0001$ & \\
\hline No & $7146(70 \%)$ & $3037(30 \%)$ & & & ref \\
\hline Yes & $98(47 \%)$ & $109(53 \%)$ & & & $2.62(1.99-3.45)$ \\
\hline Low cardiac output & & & 102.79 & $<.0001$ & \\
\hline No & $6839(71 \%)$ & $2793(29 \%)$ & & & ref \\
\hline Yes & $405(53 \%)$ & $353(47 \%)$ & & & $2.13(1.84-2.48)$ \\
\hline Tamponade & & & 36.58 & $<.0001$ & \\
\hline No & $7132(70 \%)$ & $3039(30 \%)$ & & & ref \\
\hline Yes & $112(51 \%)$ & $107(49 \%)$ & & & $2.24(1.71-2.93)$ \\
\hline Operative reexploration for bleeding & & & 35.57 & $<.0001$ & \\
\hline No & $6943(70 \%)$ & $2928(30 \%)$ & & & ref \\
\hline Yes & $301(58 \%)$ & $218(42 \%)$ & & & $1.72(1.44-2.06)$ \\
\hline Anticoagulant complications & & & 36.44 & $<.0001$ & \\
\hline No & $7153(70 \%)$ & $3053(30 \%)$ & & & ref \\
\hline Yes & $91(49 \%)$ & $93(51 \%)$ & & & $2.39(1.79-3.21)$ \\
\hline Transient stroke & & & 21.24 & $<.0001$ & \\
\hline No & $7167(70 \%)$ & $3076(30 \%)$ & & & ref \\
\hline Yes & $77(52 \%)$ & $70(48 \%)$ & & & $2.12(1.53-2.94)$ \\
\hline Permanent stroke & & & 56.13 & $<.0001$ & \\
\hline No & $7105(70 \%)$ & $3004(30 \%)$ & & & ref \\
\hline Yes & $139(49 \%)$ & $142(51 \%)$ & & & $2.42(1.91-3.06)$ \\
\hline Deep sternal infection & & & 20.54 & $<.0001$ & \\
\hline No & $7195(70 \%)$ & $3095(30 \%)$ & & & ref \\
\hline Yes & $49(49 \%)$ & $51(51 \%)$ & & & $2.42(1.63-3.59)$ \\
\hline Superficial sternal infection & & & 2.82 & .0934 & \\
\hline No & $7075(70 \%)$ & $3055(30 \%)$ & & & \\
\hline Yes & $169(65 \%)$ & $91(35 \%)$ & & & \\
\hline Leg infection & & & 15.76 & $<.0001$ & \\
\hline No & $7111(70 \%)$ & $3049(30 \%)$ & & & ref \\
\hline Yes & $133(58 \%)$ & $097(42 \%)$ & & & $1.70(1.30-2.22)$ \\
\hline Pneumonia & & & 247.51 & $<.0001$ & \\
\hline No & $6903(72 \%)$ & $2721(28 \%)$ & & & ref \\
\hline Yes & $341(46 \%)$ & $424(55 \%)$ & & & $3.15(2.72-3.66)$ \\
\hline Acute renal failure & & & 116.75 & $<.0001$ & \\
\hline No & $6957(71 \%)$ & $2855(29 \%)$ & & & ref \\
\hline Yes & $287(50 \%)$ & $291(50 \%)$ & & & $2.47(2.09-2.93)$ \\
\hline GI complications & & & 221.27 & $<.0001$ & \\
\hline No & $6869(72 \%)$ & $2716(28 \%)$ & & & ref \\
\hline Yes & $375(47 \%)$ & $430(53 \%)$ & & & $2.90(2.51-3.35)$ \\
\hline \multicolumn{6}{|l|}{ Length of stay in hospital (d) } \\
\hline Length of stay in ICU & $\begin{array}{l}\text { Median }=2 \\
\quad(Q 1, \text { Q3: 1, 5) }\end{array}$ & $\begin{array}{l}\text { Median }=3 \\
\quad(Q 1, \text { Q3: 1, 8) }\end{array}$ & $Z^{*}=14.71$ & $<.0001$ & \\
\hline Length of stay, surgery to discharge & $\begin{array}{l}\text { Median }=7 \\
\quad(\mathrm{Q} 1, \mathrm{Q} 3: 5,9)\end{array}$ & $\begin{array}{l}\text { Median }=9 \\
\quad(\mathrm{Q} 1, \mathrm{Q} 3: 7,15)\end{array}$ & $Z=34.64$ & $<.0001$ & \\
\hline Length of stay, stepdown unit & $\begin{array}{l}\text { Median }=4 \\
\quad(\mathrm{Q} 1, \mathrm{Q} 3: 2,6)\end{array}$ & $\begin{array}{l}\text { Median }=5 \\
\quad(\mathrm{Q} 1, \mathrm{Q} 3: 3,9)\end{array}$ & $Z=16.77$ & $<.0001$ & \\
\hline \multicolumn{6}{|l|}{ Mortality } \\
\hline Mortality & & & 104.58 & $<.0001$ & \\
\hline No & $6268(72 \%)$ & $2471(28 \%)$ & & & ref \\
\hline Yes & $976(59 \%)$ & $675(41 \%)$ & & & $1.75(1.57-1.96)$ \\
\hline
\end{tabular}


TABLE 2. Continued

\begin{tabular}{|c|c|c|c|c|c|}
\hline \multirow[b]{2}{*}{ Variable } & \multicolumn{2}{|c|}{ POAF } & \multicolumn{2}{|c|}{ Chi-square test } & \multirow{2}{*}{$\begin{array}{c}\text { Odds ratio } \\
\text { OR }(95 \% \text { CI })\end{array}$} \\
\hline & No, n $(\%)$ & Yes, n $(\%)$ & $\chi^{2}$ & $\boldsymbol{P}$ & \\
\hline 30-d mortality & & & 3.57 & .0587 & \\
\hline No & $6932(70 \%)$ & $2984(30 \%)$ & & & \\
\hline Yes & $312(66 \%)$ & $162(34 \%)$ & & & \\
\hline Cause of death: cardiac & & & 10.34 & .0014 & \\
\hline No & $6984(70 \%)$ & $2991(30 \%)$ & & & ref \\
\hline Yes & $260(63 \%)$ & $155(37 \%)$ & & & $1.39(1.14-1.71)$ \\
\hline Cause of death: neurologic & & & 15.02 & .0001 & \\
\hline No & $7192(70 \%)$ & $3098(30 \%)$ & & & ref \\
\hline Yes & $52(52 \%)$ & $48(48 \%)$ & & & $2.14(1.44-3.18)$ \\
\hline Cause of death: pulmonary & & & 19.57 & $<.0001$ & \\
\hline No & $7153(70 \%)$ & $3069(30 \%)$ & & & ref \\
\hline Yes & $91(54 \%)$ & $77(46 \%)$ & & & $1.97(1.45-268)$ \\
\hline Cause of death: infection & & & 5.51 & .0189 & \\
\hline No & $7194(70 \%)$ & $3110(30 \%)$ & & & ref \\
\hline Yes & $50(58 \%)$ & $36(42 \%)$ & & & $1.67(1.08-2.56)$ \\
\hline
\end{tabular}

$P O A F$, Postoperative atrial fibrillation; $O R$, odds ratio; $C I$, confidence interval; $M I$, myocardial infarction; $G I$, gastrointestinal; $I C U$, intensive care unit. *Mann-Whitney Wilcoxon test.

to a $15 \%$ higher risk of POAF. More important, the data seem to indicate that undergoing certain types of cardiac procedures carries a significant risk of POAF: An aortic or mitral valve procedure, $\mathrm{CABG}$, or left ventricular aneurysm repair introduces a $51 \%, 88 \%, 23 \%$, and $62 \%$ higher risk of POAF, respectively. Because of the small number of tricuspid valve procedures and ventricular septal defect repairs available for analysis, this study was unable to determine the POAF risk for these procedures. Other studies have reported that the incidence of POAF is greater for combined procedures than for isolated procedures. ${ }^{2}$ In this database, $8.5 \%$ of patients $(\mathrm{n}=887)$ had a combined valve and $\mathrm{CABG}$ procedure. Although this combined procedure was significant in the univariate analysis (Table 1), it was not a significant predictor in the multivariate analysis.

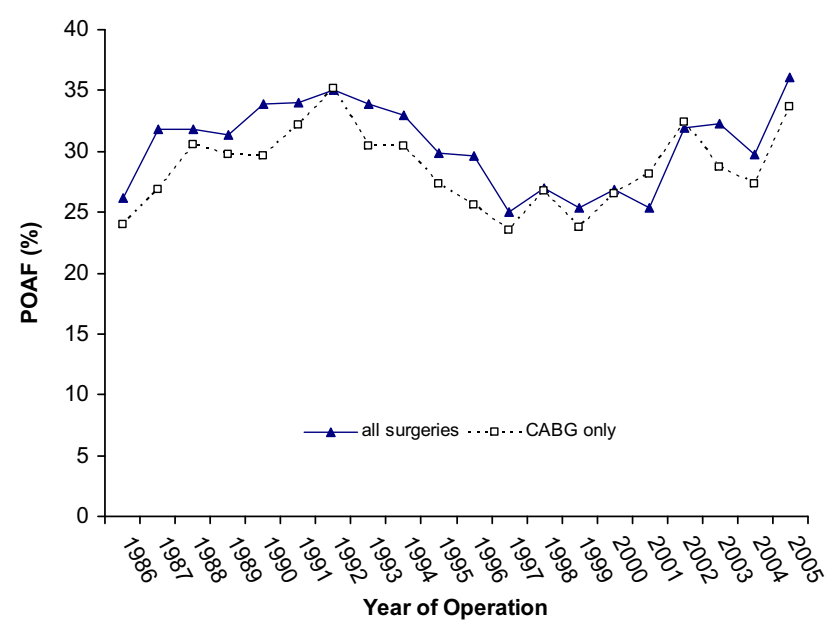

FIGURE 1. Incidence of new-onset POAF for all patients and only patients undergoing CABG over a 20-year period. $P O A F$, Postoperative atrial fibrillation; $C A B G$, coronary artery bypass grafting.
Increasing the crossclamp time during a procedure did not significantly increase the risk of POAF. Multivariate analysis also indicated that the use of intraoperative cardioplegia to arrest the heart and intraaortic balloon pump increased the risk of POAF by $23 \%$ and $27 \%$, respectively. However, the POAF risk related to intraoperative cardioplegia may have been affected by the heterogeneous nature of its reference group, which included off-pump CABG (which might have been related to less POAF), type A aortic dissection repairs where circulatory arrest was used, and other surgeries, each of which did not involve cardioplegia but carried varying risks of POAF.

Approximately $80 \%$ of the cardiac procedures involved $\mathrm{CABG}$, and more than $68 \%$ of all surgeries were lone CABG procedures. Model 2 analyzed the lone CABG surgeries and revealed that increasing age and history of CHF were the only 2 independent risk factors associated with

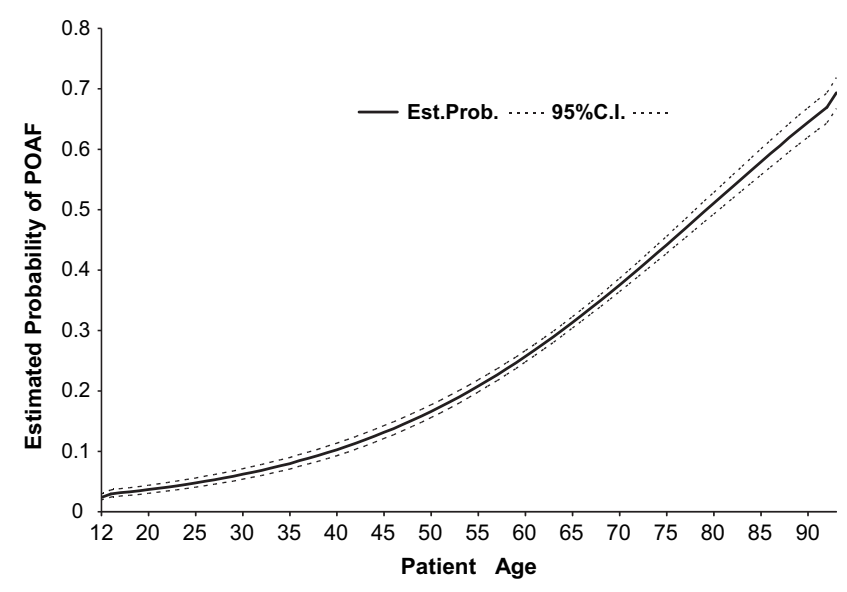

FIGURE 2. Estimated probability of POAF by age. $P O A F$, Postoperative atrial fibrillation; $C I$, confidence interval. 
TABLE 3. Predictors of new-onset postoperative atrial fibrillation and odds ratios $(95 \%$ confidence interval)

\begin{tabular}{|c|c|c|c|}
\hline Predictor & $\begin{array}{c}\text { Model 1 } \\
\text { All procedures } \\
(\mathbf{N}=\mathbf{5 0 7 5}) \\
\text { OR }(\mathbf{9 5} \% \mathbf{C I}) \\
\end{array}$ & $\begin{array}{c}\text { Model } 2 \\
\text { CABG only } \\
(\mathbf{N}=\mathbf{3 0 5 8}) \\
\text { OR }(\mathbf{9 5} \% \mathbf{C I}) \\
\end{array}$ & $\begin{array}{c}\text { Model } 3 \\
\text { Re-test of model } 1 \\
(\mathbf{N}=\mathbf{1 0 , 3 7 1 )} \\
\text { OR }(\mathbf{9 5} \% \mathbf{C I}) \\
\end{array}$ \\
\hline \multicolumn{4}{|l|}{ Demographics } \\
\hline \multicolumn{4}{|l|}{ Age group } \\
\hline $12-54$ y & ref & ref & ref \\
\hline $55-63 \mathrm{y}$ & $1.84(1.49-2.27)$ & $1.77(1.33-2.36)$ & $2.12(1.84-2.46)$ \\
\hline $64-71$ y & $3.39(2.77-4.14)$ & $3.28(2.50-4.31)$ & $3.72(3.24-4.29)$ \\
\hline $72+y$ & $4.36(3.59-5.29)$ & $4.19(3.20-5.49)$ & $5.34(4.64-6.14)$ \\
\hline \multicolumn{4}{|l|}{ Race } \\
\hline African-American & ref & & ref \\
\hline Caucasian & $1.50(1.23-1.83)$ & & $1.51(1.30-1.76)$ \\
\hline Other & $0.89(0.46-1.71)$ & & $1.12(0.72-1.73)$ \\
\hline \multicolumn{4}{|l|}{ Preoperative medical history } \\
\hline \multicolumn{4}{|l|}{ CHF } \\
\hline No & ref & ref & ref \\
\hline Yes & $1.39(1.22-1.58)$ & $1.46(1.24-1.72)$ & $1.28(1.16-1.42)$ \\
\hline \multicolumn{4}{|l|}{ Hypertension } \\
\hline No & ref & & ref \\
\hline Yes & $1.15(1.00-1.32)$ & & $1.15(1.04-1.26)$ \\
\hline \multicolumn{4}{|l|}{ Intraoperative conditions } \\
\hline \multicolumn{4}{|l|}{ Aortic valve procedure } \\
\hline No & ref & $-*$ & ref \\
\hline Yes & $1.51(1.26-1.81)$ & & $1.52(1.34-1.72)$ \\
\hline \multicolumn{4}{|l|}{ Mitral valve procedure } \\
\hline No & ref & - & ref \\
\hline Yes & $1.88(1.54-2.30)$ & & $1.91(1.64-2.23)$ \\
\hline \multicolumn{4}{|l|}{ CABG } \\
\hline No & ref & - & \\
\hline Yes & $1.23(1.03-1.47)$ & & \\
\hline \multicolumn{4}{|l|}{ LVA repair } \\
\hline No & ref & - & ref \\
\hline Yes & $1.62(1.12-2.34)$ & & $1.57(1.15-2.13)$ \\
\hline \multicolumn{4}{|l|}{ Cardioplegia used } \\
\hline No & ref & & ref \\
\hline Yes & $1.27(1.02-1.47)$ & & $1.36(1.15-1.60)$ \\
\hline \multicolumn{4}{|l|}{ IABP used } \\
\hline No & ref & & ref \\
\hline Yes & $1.24(1.04-1.49)$ & & $1.28(1.11-1.46)$ \\
\hline Goodness-of-fit: $\chi^{2}(d f) ; P$ & $7.44(8) ; P=.4901$ & $1.77(6) ; P=.9399$ & $9.83(8) ; P=.2770$ \\
\hline Likelihood ratio: $\chi^{2}(d f) ; P$ & $510.14(13) ; P<.0001$ & 186.55 (4); $P<.0001$ & $1054.41(12) ; P<.0001$ \\
\hline AUC & .69 & .65 & .70 \\
\hline
\end{tabular}

$C I$, Confidence interval; $O R$, odds ratio; $C H F$, congestive heart failure; $C A B G$, coronary artery bypass graft; $L V A$, left ventricular aneurysm; $I A B P$, intraaortic balloon pump; $A U C$, area under the curve. *Not applicable for analysis.

a higher probability of POAF. Patients with a history of CHF were $46 \%$ more likely to have POAF. In contrast with the results of model 1, being of Caucasian descent or having preexisting hypertension did not increase the risk of POAF after lone CABG procedures.

Model 3 imposed the 10 variables as predictors on a larger number of patients $(\mathrm{N}=10,371)$ and confirmed the stability of model 1's results. CABG was the only variable (in model 1) that turned out to be nonsignificant in the re-test (model 3 ). During the 20-year period examined, the percentage of patients with CHF increased from $25 \%$ to $40 \%$ (Figure 3 ). The percentage of patients with hypertension increased from $52 \%$ to $72 \%$. The use of cardioplegia decreased from $96 \%$ to $82 \%$. The percentage of patients undergoing CABG procedures decreased from $90 \%$ to $55 \%$, whereas the percentage of mitral valve and aortic valve procedures increased from $4 \%$ and $9 \%$ to $20 \%$ and $26 \%$, respectively.

The Hosmer-Lemeshow test and AUC showed that both models 1 and 3 were similar in their goodness of fit and adequate discriminative power $(\mathrm{AUC}=0.70)$. Even with 

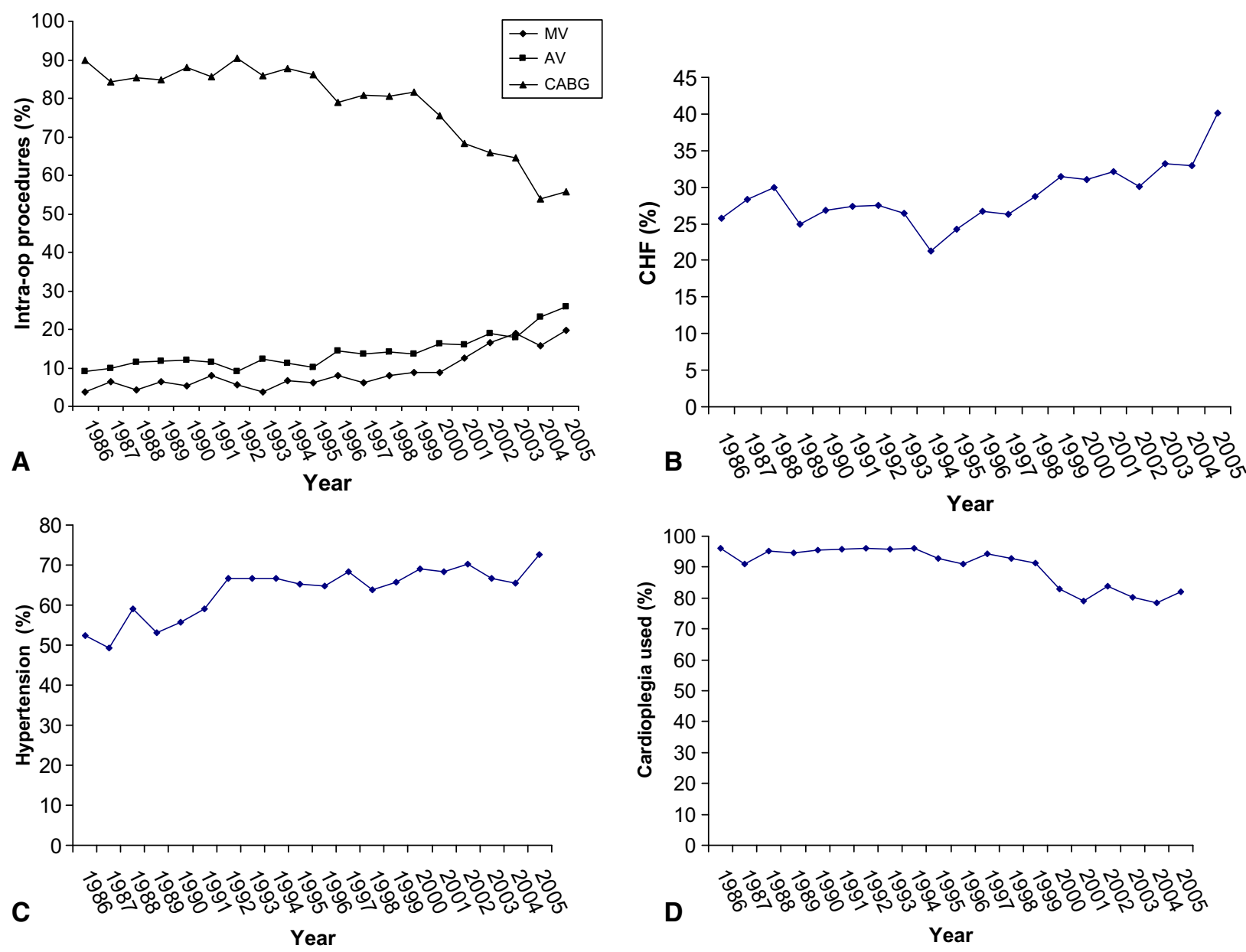

FIGURE 3. A, Trends in type of operation. B, Trend in CHF. C, Trend in hypertension. D, Trend in the use of cardioplegia. $M V$, Mitral valve; $A V$, aortic valve; $C A B G$, coronary artery bypass grafting; $C H F$, congestive heart failure.

fewer predictors, model 3 interpreted the data as well as model 1. A model with only age as a predictor had an AUC of 0.68 , illustrating the dominant effect of age on the predictability of POAF.

\section{DISCUSSION}

Logistic regression modeling is a useful tool in searching for protective and risk factors for POAF. The results of this method, however, are greatly influenced by the size and characteristics of patient samples and the spectra of factors controlled for in the analysis. The results from this study are comparatively accurate and replicable because they arose from analysis of a comprehensive group of risk factors based on a large population of patients undergoing various cardiac surgical procedures across a 20 -year time period. The incidences of POAF from observational studies from the 1980s and contemporary studies are consistent with the findings of the present study. ${ }^{2,10-13}$ The incidence of POAF oscillated between $25 \%$ and $35 \%$ over the 20 -year period of the current study. Peaks and valleys in the incidence (in Figure 1, eg, the incidence was $36 \%$ in 2005 and $24 \%$ in
1997) can be explained by the types of operations performed during a particular year and by patient demographics, according to the MLR results (Table 3). For example, valve procedures have the highest risk of POAF (OR $=1.91$ for mitral and 1.52 for aortic procedures). Among the patients in $2005,20 \%$ had mitral and $26 \%$ had aortic valve procedures, compared with only $6 \%$ and $14 \%$, respectively, in 1997 . The combination of types of surgery and patient characteristics probably explains the wide range of POAF incidences $(11 \%-50 \%)$ reported by other studies. From 1986 to 2005, the annual percentage of patients with aortic or mitral valve procedures, CHF, and hypertension increased by $17 \%$, $16 \%, 14 \%$, and $20 \%$, respectively. Considering that these factors were associated with a significantly higher risk of POAF, while the average incidence of POAF remained approximately $30 \%$, it is reasonable to conclude that we have made some progress over time in preventing POAF.

Increasing age remains strongly associated with POAF. The incidence of POAF reported in previous studies has ranged from $11 \%$ to $50 \%,{ }^{1}$ possibly because of variation in the ages of the different study groups used. In the present 
study, the estimated probability of POAF was less than $20 \%$ for patients aged less than 55 years, but increased rapidly for patients aged 55 years and more, up to a maximum of $80 \%$ (Figure 2), indicating a nonlinear relationship between age and probability of having POAF. Aging has been associated with remodeling of the atria. ${ }^{14}$ In particular, one proarrhythmic change is an increase in fibrous tissue between myocytes, which develops with age and is increased by various pathologies. This slows conduction and makes it more heterogeneous, increasing the inducibility of atrial fibrillation.

Among the 51 independent variables studied, 31 were associated with POAF based on univariate analysis (Table 1). However, multivariate analysis identified 9 factors that were associated with a higher risk of POAF: increasing age, Caucasian race, a history of CHF, preexisting hypertension, use of intraoperative cardioplegia or an intraaortic balloon pump, and 3 types of cardiac surgical procedures (Table 3 ). Knowing the factors that are associated with a greater risk of POAF will help direct research and therapeutic efforts toward the patients who need it most. For instance, an estimate based on model 3 indicates that the chance of developing POAF after an aortic valve procedure for a patient aged less than 55 years is $43 \%$. The calculated risk would be $76 \%$ for a mitral valve procedure involving cardioplegia performed in a 72-year-old Caucasian patient with a history of $\mathrm{CHF}$ and hypertension. High-risk patients could be candidates for attempted drug prophylaxis or other experimental interventions.

There has also been interest in factors associated with a decreased risk of POAF. For example, smoking has been correlated with a lower risk of $\mathrm{POAF}^{2}$ (the exact reason for this is unknown), patients who have undergone previous cardiac surgeries without experiencing POAF seem less likely to have POAF after subsequent cardiac surgeries (as indicated by the univariate analyses in this study), and pharmacologic prophylaxis with certain drugs is believed to protect against POAF. ${ }^{1,7-9,15-17}$ The present study did not find evidence, on multivariate analysis, supporting an association between any of these factors and a decreased risk of POAF.

The preoperative use of drugs, such as beta-blockers, has been inconsistently reported to have either a protective ${ }^{7,18,19}$ or no effect against POAF. ${ }^{10,13,20}$ None of the 13 medications in this study, when taken preoperatively, were found to be related to the onset of POAF. There are several possible explanations for this. First, these drugs are not definitively known to be effective against and were not prescribed to treat atrial fibrillation (patients with preexisting atrial fibrillation were excluded from the study), so it is not surprising that they were not protective against POAF. Second, the dosages and lengths of time patients were taking preoperative medications varied. Third, the fact that a patient was receiving a specific drug indicated that he/she may have had a comorbid condition requiring treatment with that drug.
In such situations, it is difficult to separate the effects of the comorbid condition from those of the drug on the onset of POAF, despite the use of multivariate analysis. Fourth, it was common for patients to be taking multiple medications, complicating the association of any single medication's effect with POAF. For these reasons, studies on the effects of pharmacologic prophylaxis for POAF should have stringently controlled experimental designs. It should also be pointed out that postoperative medications were not included in our analyses because we did not have adequate information on when these medications were given postoperatively (eg, before or after POAF developed in patients). It is possible that 1 or more of the 13 categories of medications studied may be associated with a reduced incidence of POAF when given preoperatively and continued during the immediate postoperative period.

The discriminative power of the models, as measured by the AUC, is only fair and suggests that the underlying causes and mechanisms of POAF have yet to be determined. Clearly, the strong relationship of age with POAF suggests a need for a greater understanding of the effects of aging on the underlying substrates for POAF. Limitations in data availability did not allow us to test all important factors that have reportedly been associated with POAF, for example, the echocardiographic variables quantifying left atrial and ventricular dimensions or function, the preoperative use of amiodarone, on- versus off-pump surgery, the type of incision, and the inflammatory response to surgery (including postoperative pericarditis and markers such as interleukin-6, C-reactive protein, the neutrophil/lymphocyte ratio, and plasminogen activator inhibitor- $1^{10,20-25}$ ). These limitations emphasize the need for a more researchfriendly centralized patient database with standardization of commonly investigated variables and their definitions.

\section{CONCLUSIONS}

The incidence of POAF over a 20-year period has not significantly decreased in this single-institution study. Over this study period, the mean incidence of POAF has persisted at approximately $30 \%$. This is consistent with data from other institutions showing that the incidence reported in the 1980s has remained unchanged in recent observational studies, despite the fact that numerous studies have shown a substantial reduction in incidence after various drug therapies. It is unclear why these have not translated to clinical practice. Possibly, there is a loss of efficacy when these therapies are put into a standardized clinical pathway and applied to all patients. ${ }^{13}$ In this study, antiarrhythmic drugs, when administered preoperatively, were not associated with a significant reduction in POAF. Logistic regression models using traditional preoperative and intraoperative risk factors do not adequately predict POAF. Understanding the underlying pathophysiology of POAF remains elusive, and until there is a better understanding of these mechanisms, it is 
unlikely that risk factors that adequately predict POAF will be identified or that therapies will be developed to treat this condition.

\section{References}

1. Ommen SR, Odell JA, Stanton MS. Atrial arrhythmias after cardiothoracic surgery. N Engl J Med. 1997;336:1429-34.

2. Creswell LL, Schuessler RB, Rosenbloom M, Cox JL. Hazards of postoperative atrial arrhythmias. Ann Thorac Surg. 1993;56:539-49.

3. Aranki SF, Shaw DP, Adams DH, Rizzo RJ, Couper GS, VanderVliet M, et al. Predictors of atrial fibrillation after coronary artery surgery: current trends and impact on hospital resources. Circulation. 1996;94:390-7.

4. Hashimoto K, Ilstrup DM, Schaff HV. Influence of clinical and hemodynamic variables on risk of supraventricular tachycardia after coronary artery bypass. J Thorac Cardiovasc Surg. 1991;101:56-65.

5. Leitch JW, Thomson D, Baird DK, Harris PJ. The importance of age as a predictor of atrial fibrillation and flutter after coronary artery bypass grafting. $J$ Thorac Cardiovasc Surg. 1990;100:338-42.

6. SAS Institute Inc, SAS/STAT User's Guide, Version 8, Cary, NC: SAS Institute Inc.

7. Kowey PR, Taylor JE, Rials SJ, Marinchak RA. Meta-analysis of the effectiveness of prophylactic drug therapy in preventing supraventricular arrhythmia early after coronary artery bypass grafting. Am J Cardiol. 1992;69:963-5.

8. Bradley D, Creswell LL, Hogue CW, Epstein AE, Prystowsky EN, Daoud EG. Pharmacologic prophylaxis: American College of Chest Physicians guidelines for the prevention and management of postoperative atrial fibrillation after cardiac surgery. Chest. 2005;128:39-47.

9. Mathew JP, Fontes ML, Tudor IC, Ramsay J, Duke P, Mazer CD, et al. A multicenter risk index for atrial fibrillation after cardiac surgery. JAMA. 2004;291: 1720-9.

10. Gibson PH, Cuthbertson BH, Croal BL, Rae D, El-Shafei H, Gibson G, et al. Usefulness of neutrophil/lymphocyte ratio as predictor of new-onset atrial fibrillation after coronary artery bypass grafting. Am J Cardiol. 2010;105:186-91.

11. Saso S, Vecht JA, Rao C, Protopapas A, Ashrafian H, Leff D, et al. Statin therapy may influence the incidence of postoperative atrial fibrillation. Tex Heart Inst J. 2009;36:521-9.

12. Mauermann WJ, Nuttall GA, Cook DJ, Hanson AC, Schroeder DR, Oliver WC. Hemofiltration during cardiopulmonary bypass does not decrease the incidence of atrial fibrillation after cardiac surgery. Anesth Analg. 2010;110:329-34.

13. Kim MH, Deeb GM, Morady F, Bruckman D, Hallock LR, Smith KA, et al. Effect of postoperative atrial fibrillation on length of stay after cardiac surgery (The postoperative atrial fibrillation in cardiac surgery study [PACS2]). Am J Cardiol. 2001;87:881-5.

14. Pandit SV, Jalife J. Aging and atrial fibrillation research: where we are and where we should go. Heart Rhythm. 2007;4:186-7.

15. Hogue CW, Creswell LL, Gutterman DD, Fleisher LA. Epidemiology, mechanisms, and risks: American College of Chest Physicians guidelines for the prevention and management of postoperative atrial fibrillation after cardiac surgery. Chest. 2005;128:9-16.

16. Burgess DC, Kilborn MJ, Keech AC. Interventions for prevention of postoperative atrial fibrillation and its complications after cardiac surgery: a meta-analysis. Eur Heart J. 2006;27:2846-57.

17. Mariscalco G, Lorusso R, Klersy C, Ferrarese S, Tozzi M, Vanoli D, et al. Observational study on the beneficial effect of preoperative statins in reducing atrial fibrillation after coronary surgery. Ann Thorac Surg. 2007;84:1158-65.

18. Frost L, Molgaard H, Christiansen EH, Hjortholm K, Paulsen PK, Thomsen PEB. Atrial fibrillation and flutter after coronary artery bypass surgery: epidemiology, risk factors and preventive trials. Int J Cardiol. 1992;36:253-61.

19. Andrews TC, Reimold SC, Berlin JA, Antman EM. Prevention of supraventricular arrhythmias after coronary artery bypass surgery: a meta-analysis of randomized control trails. Circulation. 1991;84(Suppl III):236-43.

20. Pretorius M, Donahue BS, Yu C, Greelish JP, Roden DM, Brown NJ. Plasminogen activator inhibitor-1 as a predictor of postoperative atrial fibrillation after cardiopulmonary bypass. Circulation. 2007;116(Suppl I):1-7.

21. Fuster V, Ryden LE, Cannom DS, Crijns HJ, Curtis AB, Ellenbogen KA, et al. ACCAHAESC 2006 guidelines for the management of patients with atrial fibrillation - a report of the American College of Cardiology/American Heart Association Task Force on Practice Guidelines and the European Society of Cardiology Committee for Practice Guidelines (Writing Committee to Revise the 2001 Guidelines for the Management of Patients With Atrial Fibrillation). J Am Coll Cardiol. 2006;48:e149-246.

22. Daoud EG, Strickberger SA, Man KC, Goyal R, Deeb GM, Bolling SF, et al. Preoperative amiodarone as prophylaxis against atrial fibrillation after heart surgery. N Engl J Med. 1997;337:1785-91.

23. Guarnieri T, Nolan S, Gottlieb SO, Dudek A, Lowry DR. Intravenous amiodarone for the prevention of atrial fibrillation after open heart surgery: the Amiodarone Reduction in Coronary Heart (ARCH) trial. J Am Coll Cardiol. 1999; 34:343-7.

24. Ishida K, Kimura F, Imamaki M, Ishida A, Shimura H, Kohno H, et al. Relation of inflammatory cytokines to atrial fibrillation after off-pump coronary artery bypass grafting. Eur J Cardiothorac Surg. 2006;29:501-5.

25. Lo B, Fijnheer R, Nierich AP, Bruins P, Kalkman CJ. C-reactive protein is a risk indicator for atrial fibrillation after myocardial revascularization. Ann Thorac Surg. 2005;79:1530-5. 